Editorial

\title{
More than meets the eye - an association between diet soft drink consumption and proliferative diabetic retinopathy
}

\author{
Peter van Wijngaarden PhD FRANZCO ${ }^{1,2,3}$ and Stuart Keel PhD ${ }^{1}$
}

1. Centre for Eye Research Australia, University of Melbourne, Royal Victorian Eye and Ear Hospital, Melbourne, Australia.

2. Ophthalmology, University of Melbourne, Department of Surgery, Melbourne, Australia

3. Royal Victorian Eye and Ear Hospital, East Melbourne, Australia.

Correspondence: Peter van Wijngaarden, Centre for Eye Research Australia, University of Melbourne, Royal Victorian Eye and Ear Hospital, Melbourne, Australia peterv@unimelb.edu.au

Conflict of interest: None

Funding sources: None

This is the author manuscript accepted for publication and has undergone full peer review but has not been through the copyediting, typesetting, pagination and proofreading process, which may lead to differences between this version and the Version of Record. Please cite this article as doi: $10.1111 /$ ceo.13377

This article is protected by copyright. All rights reserved. 
"Things are not always as they seem; the first appearance deceives many". This ancient wisdom, attributed to the Roman fabulist Phaedrus, has been proven correct time and again in human history. The growing popularity of diet soft drinks in recent years has been driven by increasing recognition of the adverse health consequences of sugar consumption and, in particular, the potential harms of sugar-sweetened soft drinks. Diet soft drinks containing artificial sweeteners in place of sugar, have been marketed as a healthier choice - lower in carbohydrates and energy. "Regret nothing - no sugar, no calories" is a recent marketing catch-phrase of a leading diet soft drink. Consumers have responded to these implicit health messages as well as economic imperatives, such as the taxes on sugar-sweetened beverages that are enforced in many countries, and diet beverages now make up a substantial proportion of the global soft drink market. ${ }^{1}$ Furthermore, owing to their less acute effects on blood glucose levels, dietary advice for people with diabetes identifies diet soft drinks as a healthier alternative to sugar-sweetened beverages. ${ }^{2,3}$ An interesting study by Fenwick et al., in this edition of the Journal, highlights the potential harm of diet soft drink consumption in people with diabetes. ${ }^{4}$

The cross-sectional study of 609 people with diabetes (87.5\% type 2 diabetes) investigated the association between soft drink consumption and diabetic retinopathy (DR). Individuals who consumed more than four cans of diet soft drink per week (heavy consumption) had more than a two-fold increased likelihood of having proliferative $D R(O R=2.62)$ than those who did not. Diet soft drink consumption was independently associated with this risk in multivariate analyses. Interestingly, the consumption of sugar-sweetened soft drink was not associated with DR in this study.

Studies of the associations between soft drink consumption and DR are scarce and the investigators are to be commended for their work. Notable strengths of the 
study include the inclusion of a wide range of known and potential risk factors for DR and their use of a well-validated food frequency questionnaire to ascertain diet, as well as a standardised protocol to grade retinal images.

While the study provides important evidence in support of an association between diet soft drink consumption and retinopathy, further studies are warranted to interrogate this link. As acknowledged by the authors, cross-sectional studies have inherent limitations that preclude the attribution of causation. It is notable that data for soft drink consumption were missing for $21 \%$ of subjects in the study. While robust statistical methods were used to compensate for missing data, scope for under- or over-estimation of the strength of the observed association remains. In addition, although a range of known risk factors for DR were included in the analysis, the interaction of soft drink consumption with other dietary variables may confound the observed association. As a case in point, artificial sweeteners are commonly used by people with diabetes in foods other than diet soft drinks, and it would be interesting to assess whether the association stands when other sources of artificial sweetener are accounted for. The absence of an association between diet soft drink consumption and less advanced stages of retinopathy also warrants attention, as a dose-response relationship might be expected, given that nonproliferative retinopathy usually precedes proliferative disease.

Fenwick and colleagues highlight the related findings of other studies that have identified associations between diet soft drink and cardiovascular disease. ${ }^{5,6}$ While little is known about the mechanisms underlying these disease associations, it may be that diet soft drink consumption drives other dietary behaviours. We are beginning to understand the profound influence of the gastrointestinal microbiome on metabolism and disease and it may be that dietary sweeteners affect gut flora to the detriment of systemic vascular health., 
Research often generates as many questions as it answers. The paper by Fenwick et al. underscores the need for further studies to delineate the influence of dietary soft drink consumption on the risk of DR and health. There may well be more to these beverages than meets the eye. 


\section{REFERENCES}

1. Bonnet C, Requillart V. Does the EU sugar policy reform increase added sugar consumption? An empirical evidence on the soft drink market. Health economics 2011; 20(9): 1012-24.

2. ADA. Low-Calorie Sweeteners. 2014 (accessed 26th June 2018).

3. NSW D. Food choices for people with diabetes. 2012 (accessed 26th June 2018).

4. Fenwick EK, Gan ATL, Man REK et al. Diet soft drink is associated with increased odds of proliferative diabetic retinopathy Clin Experiment Ophthalmo/2018; 46: pppp

5. Azad MB, Abou-Setta AM, Chauhan BF, et al. Nonnutritive sweeteners and cardiometabolic health: a systematic review and meta-analysis of randomized controlled trials and prospective cohort studies. Cmaj 2017; 189(28): E929-e39.

6. Gardener H, Rundek T, Markert M, Wright CB, Elkind MS, Sacco RL. Diet soft drink consumption is associated with an increased risk of vascular events in the Northern Manhattan Study. Journal of general internal medicine 2012; 27(9): 11206.

7. Frankenfeld CL, Sikaroodi M, Lamb E, Shoemaker S, Gillevet PM. Highintensity sweetener consumption and gut microbiome content and predicted gene function in a cross-sectional study of adults in the United States. Annals of epidemiology 2015; 25(10): 736-42.e4.

8. Suez J, Korem T, Zilberman-Schapira G, Segal E, Elinav E. Non-caloric artificial sweeteners and the microbiome: findings and challenges. Gut microbes 2015; 6(2): 149-55. 


\section{University Library}

\section{- $\mathrm{M}$ IIN E R VA A gateway to Melbourne's research publications}

Minerva Access is the Institutional Repository of The University of Melbourne

\section{Author/s:}

van Wijngaarden, P;Keel, S

Title:

More than meets the eye: an association between diet soft drink consumption and proliferative diabetic retinopathy

\section{Date:}

2018-09-01

\section{Citation:}

van Wijngaarden, P. \& Keel, S. (2018). More than meets the eye: an association between diet soft drink consumption and proliferative diabetic retinopathy. CLINICAL AND EXPERIMENTAL OPHTHALMOLOGY, 46 (7), pp.719-720. https://doi.org/10.1111/ceo.13377.

Persistent Link:

http://hdl.handle.net/11343/284603 